\title{
COLLECTIVE APPROACH FOR MANETS TO SUPPORT PACKET LOSS AND DELAY SENSITIVE APPLICATIONS IN REAL
}

\author{
Anuradha Patil ${ }^{1}$, Sridhar $K^{2}$ \\ ${ }^{1}$ PDA College of Engineering, Gulbarga, Karnataka India \\ ${ }^{2}$ PDA College of Engineering, Gulbarga, Karnataka India
}

\begin{abstract}
Mobile ad hoc network is network with different applications are developing with different service requirement. In particular multimedia applications and other real-time applications e.g. voice transmission. MANET is a autonomous network where communicating entities can communicate without the aid of any downlink power controller called base stations. Therefore there is no centralized control over links. Further the link quality varies due to mobility of the nodes. Existing routing protocols like AODV or any other QoS routing does not account for changes in link quality once the path is established. Therefore the performance of such a system degrades with high mobility. Hence in this work we emphasis on incorporating link quality estimation based on mobility prediction along with Node's geographic location and incorporate same in the routing decision further new routes are accumulated and the primary transmission path is changed in case a better route in terms of link quality is obtained. We mingled the idea of link stability and power consumption with Location aware routing to propose a new technique for MANET that reduces control overhead and provides better performance.
\end{abstract}

\section{INTRODUCTION \& BACKGROUND}

A typical text on Mobile Ad hoc NET works (MANETS) will describe such networks as simply being "a collection of mobile nodes, communicating among themselves over wireless links and thereby forming a dynamic, arbitrary graph" - listing wireless characteristics and graph dynamics as the main challenges for designing protocols and applications for this network.

A MANET is an Independent Basic Service Set (IBSS). Ad hoc is a Latin term and literally means "for this purpose only." In other words, it is an autonomous collection of mobile nodes, with networks built on the fly for a specific purpose (i.e., emergency situations, rescue operations, battlefield situations, etc.), that talk to each other over bandwidth constrained wireless links.

Despite their challenging aspect, MANET can be deployed at low cost in austere areas with no pre-existing infrastructure. MANET is an infrastructure-less network; i.e., there is no centralized server. Each host in this network can act as a router and contribute to the routing process. Since the nature of the network is mobile, the topology keeps changing and is highly unpredictable. An efficient routing protocol is to be designed to deal with this ever-changing topology. It will provide functionality such as route discovery, link failure detection, and notification, offering very low packet delay and high throughput to preserve QoS.

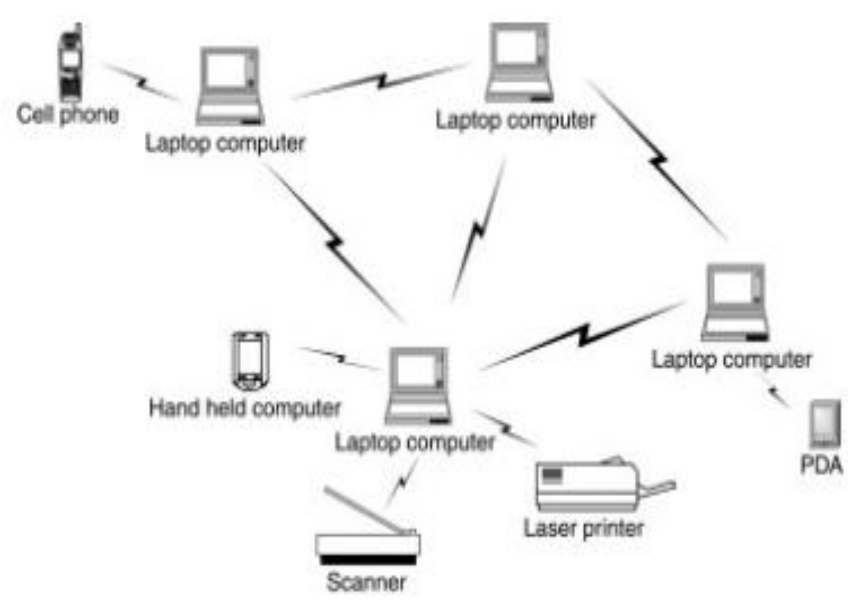

Example of MANET architecture

MANET has access gateways to provide access to the wired backbone or the internet. It is a wireless network comprising of a collection of fixed wireless gateways and mobile nodes that change their locations dynamically and organize themselves arbitrarily.

\footnotetext{
Some of the characteristics of MANETs are:

No fixed infrastructure

Peer-to-peer communication

Multi-hop routing

Frequent change in associations
} 


\subsection{Applications of MANETS}

Potential applications of MANETs are briefly addressed below.

MANETS find their applications in many fields.

Personal area networking: Used for accessing, sharing, and processing data via

The Internet

Cell phone

Laptop computers

Ear phone

Wrist watch

Military environments: To equip soldiers with devices in enemy environments so they can communicate with each other for tactical operations. Soldiers equipped with Personal Digital Assistants (PDAs)

Tanks

Planes

Civilians environments: Used for accessing and sharing data with other potential users; e.g., distribution of presentations, exchange of information, etc.

Taxi cab network

Meeting room

Sports stadiums

Small aircrafts

Emergency operations: To setup communication in lifethreatening situations.

Search and rescue

Emergency situation: policing and fire fighting

Disaster recovery

\subsection{Case1}

Let us consider as shown in figure source node want to communicate with destination node it should communicate with intermediate node. Each node having its own coverage area. Each node should broadcast the message to its neighboring node, this procedure continues till it reaches the destination. All the intermediate nodes act as a Relay nodes and each relay node having its own relay region.

Each transmission call as hop. The network should select such a route it should have minimum hop counts. That will be shortest route for transmission.

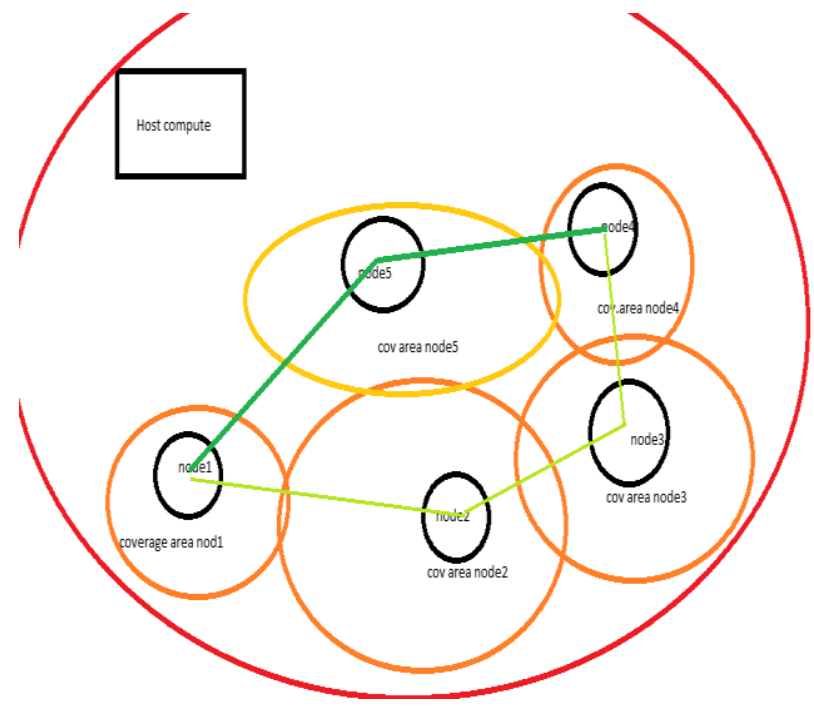

Fig 1

As shown in the figure node1 having its own coverage area as shown if node 2 comes in coverage area of node 1 than node 2 get the access, now node 3 comes in a picture if node 3 is in coverage area of node 2 that node 3 will get the access, similarly all nodes. If node1 want to send the data to node4 than should follow the path node1, node2 node 3 and finally noe 4 in this case hop counts is 3 . In mean while node 5 occurs than node1 select the route as node1, node 5 and finally node 4 so in this case the number of hop counts is 2 . So the second route is the shortest route discovery.

\subsection{Case2}

Consider the scenario as if node 2 is not in the coverage area of node 1 , than the data transmission is through host.

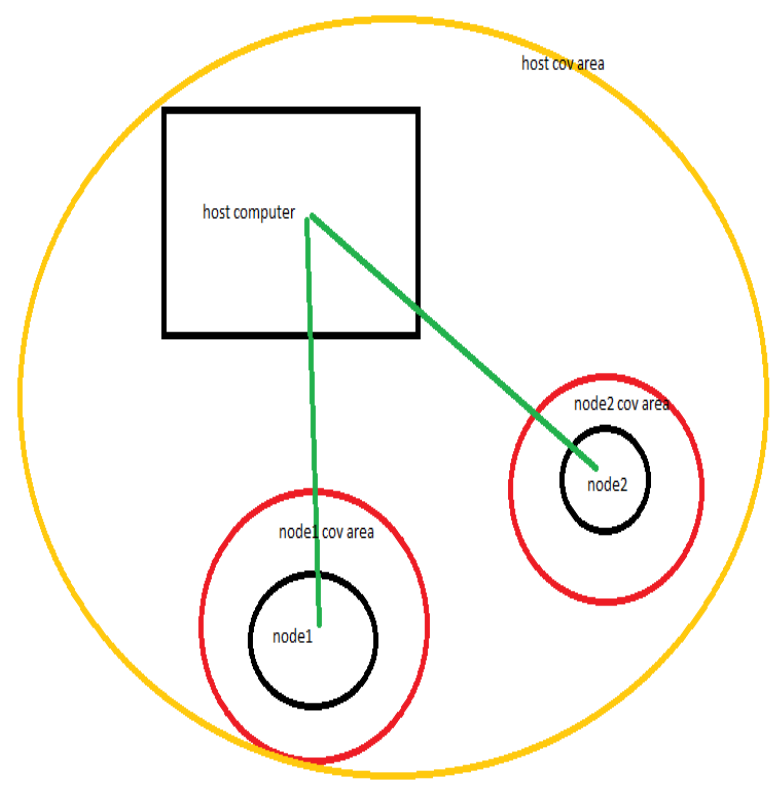


As shown in the above figures for example consider the wifi setup. If node 1 and node 2 are not in the coverage area than node1 directly cannot communicate with node2, the communication will takes place via host computer.

\subsection{Statement of the Problem}

The major problem with adhoc network is link quality variations due to mobility. Therefore in this work we propose a technique for estimating link quality between two nodes by their relative mobility and motion trajectory and incorporate same in the routing decision. This is achieved by finding appropriate relay regions between source to destination and forwarding the packets through the region.

\subsection{Objective of the Study}

The link quality is one of the great challenges in MANET which effects the transmission quality. There are several techniques for protecting against link failures and downgraded link quality like multipath routing or backup routing. Link quality is measured through power loss measurement, but the channel power loss itself depends upon the distance between the nodes. Cost between nodes provides the way to measure the link stability.

Cost depends upon the direction of transmission which in turn depends upon node's position. However as the nodes keep moving in MANET, it's position changes. The base paper does not account for future position. Hence we also incorporate this parameter of direction of movement and choose the nodes which are moving towards destination for longer link life.

If cost is low then that path is selected as highly stable. On the other if the cost is high then that particular path is not stable and hence dropped. In highly mobile environment the measured link quality therefore can vary. Hence in this work we propose a novel technique for estimating link quality by estimating the trajectory and velocity of the node movement. The objective is also to obtain fresh paths with better link quality.

\section{SYSTEM DESIGN}

\subsection{Algorithm}

1. Generate RREQ from source to destination, mentioning maximum number of paths that a node can be part of.

2. When the RREQ is received by the intermediate node, the node checks whether it is intended destination or else forwards the RREQ to next intermediate node.

3. A link stability is calculated between every nodes the path which is stable between nodes forwards the RREQ to the neighboring node.

$$
L_{s t}=\frac{r}{\sqrt{\left\{\left(x_{1}^{\prime}-x^{\prime}{ }_{2}\right)+t\left(v_{1} \cos \theta_{1}-v_{2} \cos \theta_{2}\right)\right\}^{2}}}
$$

4. As and when a node forwards the RREQ, it adds cost information along the way.

\section{Cost=Link stability $+1 /$ Power}

Power=txpower

5. Once the RREQ packets are received by destination, the destination calculates the cost as total cost accumulated in the path.

\section{$\operatorname{Cost}=\mathbf{C 1}+\mathbf{C} 2+\ldots . .+\mathrm{Cn}$}

6. Power loss is also calculated by

\section{Powerloss $=1 /$ distance ${ }^{*}$ distance}

7. Destination generates RREP for the best stable paths with minimum power loss. 


\subsection{Dataflow Diagram}

$\mathrm{x}, \mathrm{y}$, theta, $\mathrm{v}$, disp \& cost

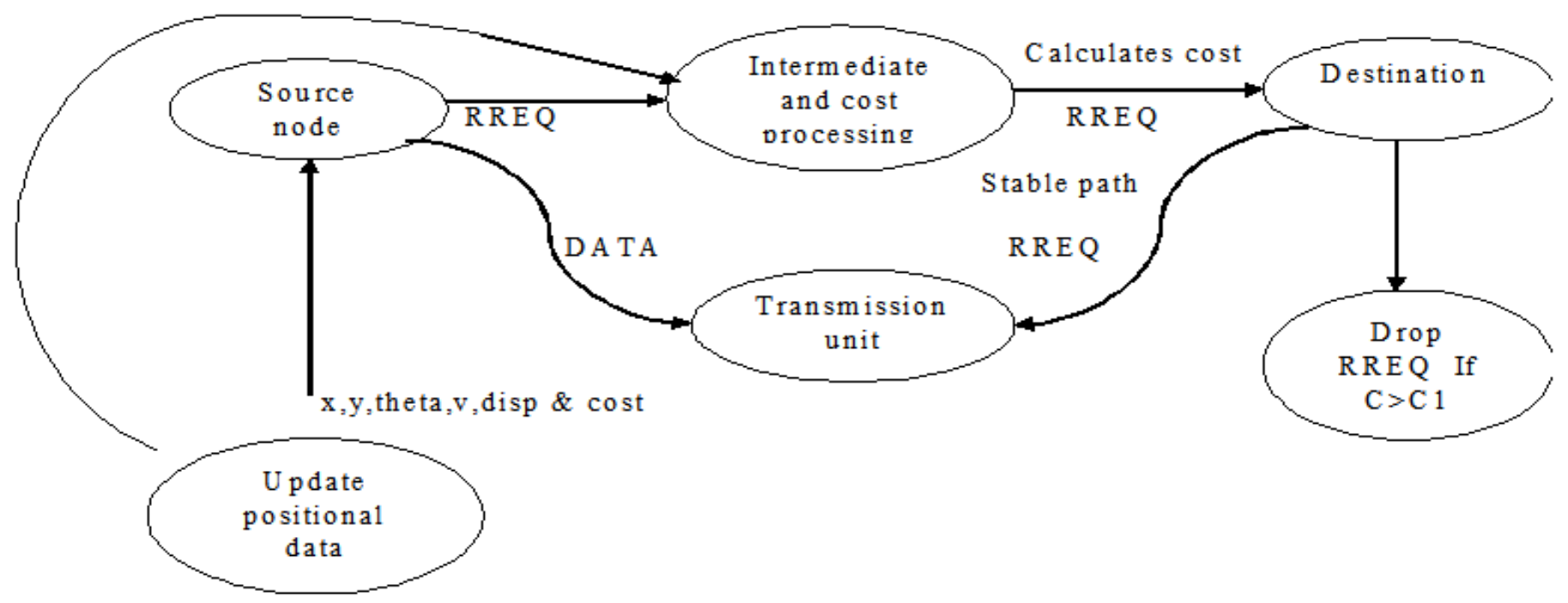

\subsection{Flow Chart}

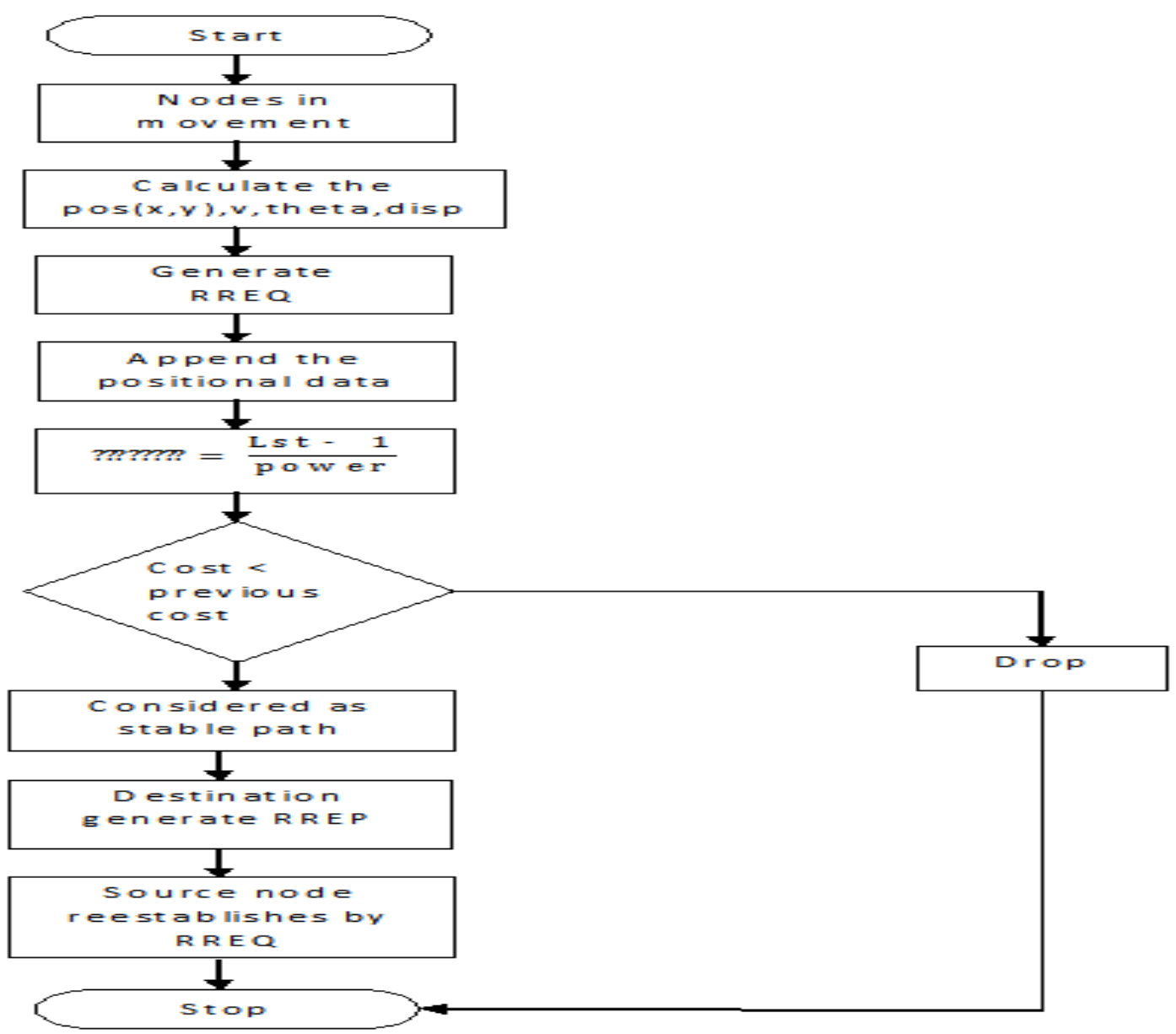




\subsection{Sequence Diagram}

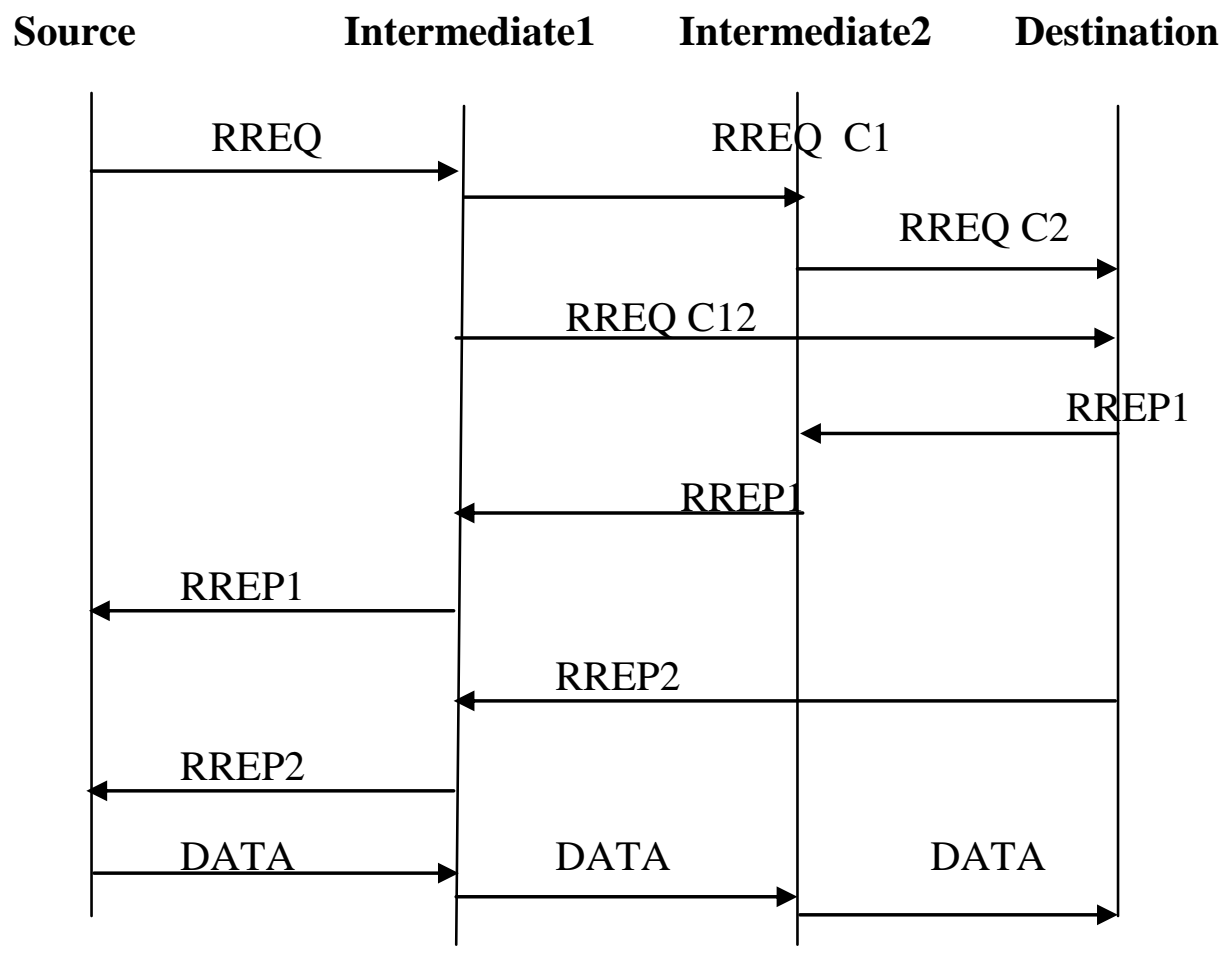

\section{IMPLEMENTATION ALGORITHM}

1) Develop the network with more number of mobile nodes. The nodes are spread over area. Allocate the mobility for the selected node. choose the speed between 2 to $3 \mathrm{~m} / \mathrm{s}$. randomly select the mobile point. Node position is represented as a point P.

Start the transmission and continue for $\mathrm{t}$ seconds.

2) Random way point:

For $\mathrm{i}=1: 1: \mathrm{N}$

a) catch the position of first point

b) Move towards assigned point speed $\mathrm{v} \mathrm{m} / \mathrm{s}$

c) Update first point

d) $\operatorname{If}(\mathrm{P}[\mathrm{m}]==\mathrm{P} 1[\mathrm{n}])$

Pause for pt milliseconds.// pt=pause time

Goto(a)

End

Update the co-ordinate values.

End

3) At MAC layer

Estimate the received power of the packet Pts.

a) if (MAC has the contention)

Forward the packets that are been sent by routing layer

Else
i)Store the packets in queue

ii) Wait for the channel access.

End

4) At Network Layer

Bypass MAC and calculate Energy directly and store in variable Ecur

a) Generate beacon packets to neighbors

b) Receive beacon packets forwarded by the neighbors

c) Prepare Routing table(ID,Hops,Energy)

d) Update neighbors table

e) If receiving node is coordinator

If coordinator has finished receiving all beacons

Generate coordinator beacon

End

Else

Increase the hop field value

Forward the packets.

End

f) If(RREQ is received)

Current node has enough energy?

Yes: Forward RREQ

Update route information

No: Drop RREQ

End

g) If(RREP is received) 
Update route information

Forward to precursor node

Notify PAN coordinator

End

h) If (a data packet is received for application)

Forward to MAC layer

Update energy

End

i) If(Control message is received from the MAC)

Generate RERR

Update energy

End

5. At Physical layer:

As packet is received .

Find type of Packet

Change the Energy level according to th equation 1

6. At the end of the simulation, calculate throughput, latency, control overhead, packet delivery ratio

\section{PERFORMANCE METRICS:}

We measure different performance metrics. In particular, for each source we evaluate:

End-to-End Delay: The time interval between the transmission of the packet by a source node and the reception at the destination.

Throughput: Network throughput is the average rate of successful message delivery over a communication channel. This data may be delivered over a physical or logical link, or pass through a certain network node. The throughput is usually measured in bits per second (bit/s or bps), and sometimes in data packets per second or data packets per time slot.

Packet loss: percentage of packets lost, as the ratio between the number of packets successfully received at the sink and all packets generated by the source node.

Control overhead: It is defined as number of control packets transmitted for every data packet delivered.

Packet Delivery Ratio: It is defined by a factor of number of packets received by number of packets transmitted.

Network Lifetime: As the nodes communicate, they lose energy. When energy is below a minimum threshold level, the node dies and cant participate in communication. The time interval from the start of simulation to the time when there exists atleast one node for communication is called network lifetime. It it's a quantitive measure for energy efficiency.

Pause Time: It is the time period between two subsequent movement in the mobility model of a node.

Node Density: It is defined as Number of Node v/s the area over the nodes are spread and gives the link density or number of nodes in average that can communicate over the area.

BER: We define ECR as the ratio of total energy spent by MAC layer packet transmission v/s energy consumption by data transmission.

\section{SIMULATION PARAMETERS}

Parameters for simulation setup are given in table 1 .

Table 1

\begin{tabular}{|l|l|}
\hline Routing & $\begin{array}{l}\text { AODV, Cross layer Energy Aware } \\
\text { AODV }\end{array}$ \\
\hline Channel Bandwidth & 250kbit/s (IEEE 802.15b) \\
\hline Channel Delay & 0.0001 sec \\
\hline Channel Error rate & 0.000001 \\
\hline Channel data rate & $250 \mathrm{kbit} / \mathrm{s}$ \\
\hline $\begin{array}{l}\text { Channel Contention } \\
\text { Mechanism }\end{array}$ & $\begin{array}{l}\text { Slotted version of CSMA/CA } \\
\text { channel access protocol. }\end{array}$ \\
\hline Node placement & Random \\
\hline Mobility Model & RWP,Rwalk \\
\hline Message packet size & 512 bytes(4096bits) \\
\hline Enabled Node & 7 \\
\hline Burst Interval & Normally Distributed(2,1,0) \\
\hline Input Buffer Size & 8.38864 e6 bits \\
\hline Radio Model & Free Space Model \\
\hline Radio Range & $100 \mathrm{~m}$ \\
\hline MAC & $\mathbf{8 0 2 . 1 5 . 4}$ \\
\hline Simulation Area & $10-100$ \\
\hline Maximum Node & 10 Seconds \\
\hline Beacon Interval & .1 second \\
\hline Beacon Monitoring Timeout & 10 b \\
\hline SNR Threshold & \\
\hline
\end{tabular}




\section{SIMULATION RESULTS}

(a) Low node density $($ node $=100)(b)$ high node density $($ node $=500)$

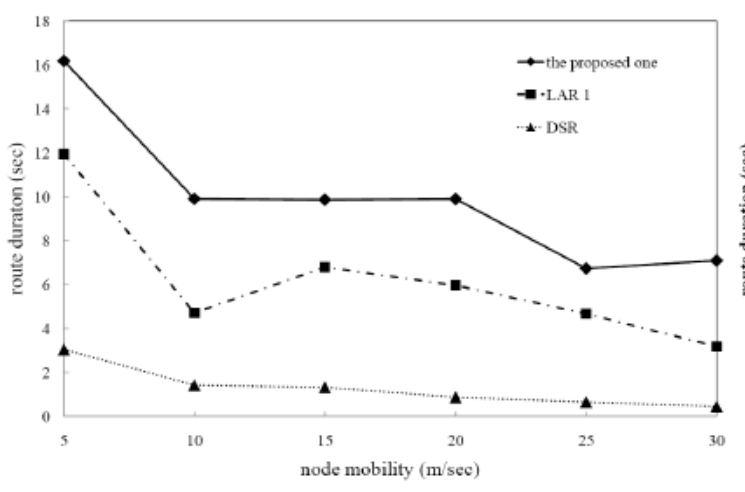

(a) low node density (node $=100)$

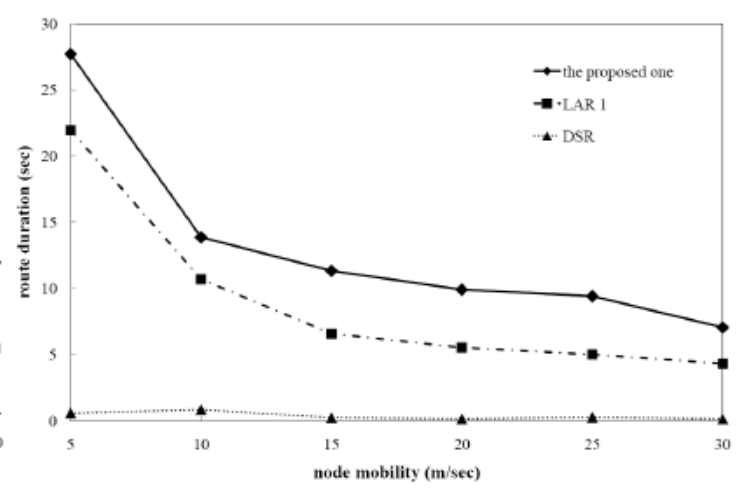

(b) high node density (node $=500$ )

Figure 9. Effect on the route duration
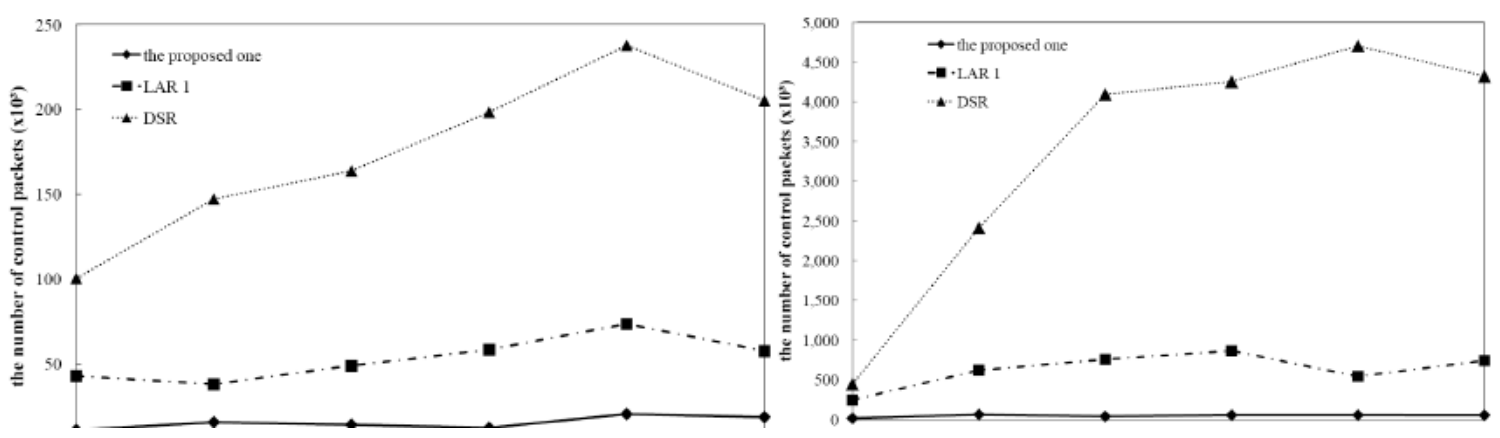

(a) Density of node at low density level (node $=99-100)($ b) at high Density level (node $=400-500)$

\section{REFERENCES}

[1]. Applications of mobile ad-hoc networks my article applications of mobile ad-hoc networks at http://www.computingunplugged.com/issues/issue2004 09/00001371001.html Humayun Bakht School of Computing and Mathematical Sciences Liverpool John Moores University Email:humayunbakht@yahoo.co.uk

[2]. A QoS architecture for MANETs supporting real-time peer-to-peer multimedia applications $\square$ Carlos T. Calafate, Juan-Carlos Cano, Pietro Manzoni, and Manuel P. Malumbres Department of Computer Engineering

[3]. QoS Multi Meshed Tree Routing in Tethered MANET.

[4]. Mobile Ad Hoc Networks Tutorial at CIT'2000 Bhubaneshwar, Dec 2023 Sridhar Iyer IIT Bombay.

[5]. An Overview of Mobile Ad Hoc Networks: Applications and Challenges Jeroen Hoebeke, Ingrid Moerman, Bart Dhoedt and Piet Demeester 